Rapid Reviews COVID-19

\title{
Review 1: "Human-organ- chip-enabled pipeline to rapidly repurpose therapeutics during viral pandemics"
}

\section{Cathryn Sundback ${ }^{1}$}

${ }^{1}$ Massachusetts General Hospital, Department of Surgery

Published on: Sep 15, 2020

DOI: 10.1162/2e3983f5.be6dd265

License: Creative Commons Attribution 4.0 International License (CC-BY 4.0). 


\section{$\underline{\text { RR:C19 Evidence Scale rating by reviewer: }}$}

- Strong. The main study claims are very well-justified by the data and analytic methods used. There is little room for doubt that the study produced has very similar results and conclusions as compared with the hypothetical ideal study. The study's main claims should be considered conclusive and actionable without reservation.

$* * * * * * * * * * * * * * * * * * * * * * * * * * * * * * * * * * * * * * * *$

\section{Review:}

The manuscript by Si et al. explores the potential of a human small airway tissue chip model to screen therapeutic compounds towards the treatment of influenza infections and the prevention of SARS-CoV-2 viral infections. Coupling existing rapid cell-based screening assays with tissue chip models has the potential to rapidly identify a small number of promising drug candidates for preclinical animal testing, which ultimately expedites drug repurposing during pandemics.

Building on their extensive expertise in microfluidic tissue chips, this research team first applied their mature physiologic small airway bilayer microfluidic model toward assessing treatments for influenza infections. This sophisticated model is based on a human differentiated lung airway epithelial basal stem cell - pulmonary microvascular endothelial cell bilayer with flow through the vascular channel and a developed airliquid interface in the epithelial channel. This team demonstrated that this highly physiologic tissue chip accurately models human airway physiology. It recapitulates tissue-tissue interfaces, recruitment of circulating immune cells, and cytokine expression as well as viral disease states. Most importantly, the tissue chip results accurately reflect the therapeutic responses of the influenza-infected epithelium to drug treatments.

The SARS-CoV-2 proof-of-concept studies screened the effectiveness of a small drug panel with antiviral properties to prevent viral entry (infection). These studies utilized in vitro and in vivo models, with the progression from rapid cell-based assays to a physiologic human small airway tissue chip platform, and finally to a preclinical hamster model. Using pseduoparticles of SARS- CoV-2, the rapid cell-based assays examined viral entry in dose-response studies while the tissue chip study was conducted with the same drugs at their human Cmax concentrations. These in vitro studies identified amodiaquine as the drug best able to limit SARS-CoV-2 infection 
prophylactically; in comparison with the controls, viral entry decreased by $55-60 \%$ for both amodiaquine and its active metabolite, desethylamodiaquine. Subsequent in vivo tests in Syrian golden hamsters demonstrated the efficacy of amodiaquine to inhibit infection prophylactically. The drug cut the lung SARS-CoV-2 viral load by $60 \%$, largely prevented the formation of pathological lung lesions similar to those observed in COVID-19 patients with pneumonia, and reduced viral transmission by $90 \%$ from SARSCoV-2 infected hamsters. Thus, this multi-screen methodology rapidly identified a drug that effectively limited SARS-CoV-2 entry into prophylactically-treated host airway and lung cells.

As an aside, this SARS-CoV-2 study highlights the importance of matching the biological properties of the rapid assay cell line to the study requirements. In the presented work, the rapid drug screen was primarily performed with Huh-7 cells, which express low levels of the ACE-2 receptor but only minimal levels of the serine protease TMPRSS2 for spike protein

priming. For efficient cell entry, the SARS-CoV-2 virus requires both transmembrane proteins, which epithelial cells of the physiologic human airway tissue chip highly express. Despite the low ACE-2/TMPRSS2 expression levels of the Huh-7 cells, the rapid cell-based assay was a fair predictor of the tissue chip-based results, except when the Cmax for a drug lay far outside the tested dose range of the rapid assay or for the drugs chloroquine and hydroxychloroquine. For these two drugs, the rapid assay and the tissue chip demonstrated opposing results with observed viral entry inhibition and no inhibition, respectively. Supporting the tissue chip results, Hoffman et al. clearly showed that SARS-CoV-2 entry required TMPRSS2. They found that choloroquine and hydroxychloroquine did not inhibit viral infection in Calu-3 and VeroTMPRSS2 cells, both of which express high levels of ACE-2 and TMPRSS2. In contrast and similar to the current study, Hoffman et al. showed that chloroquine and hydroxychloroquine did significantly prevent infection of Vero cells, which express minimal TMPRSS2 (Hoffmann, 2020).

Clearly, the cell line choice for the rapid assay impacts viral entry. Without a closer ACE- 2/TMPRSS2 profile match between the cell line and physiologic airway epithelial cells, the true significance and importance of the human airway tissue chip results remain unknown for viral infection studies. Conceivably, the use of this physiological tissue chip could lead to the discovery of additional aspects of the physiologic environment that impact SARS-CoV-2 infection. Nevertheless, this tissue chip will 
undoubtedly be required to screen viral drug treatments for SARS-CoV-2-infected epithelium.

In summary, the ability of amodiaquine to prophylactically block viral entry and prevent transmission of SARS-CoV-2 is exciting. However, further tests are necessary to assess the effectiveness of amodiaquine to treat in vitro and in vivo infected cells and tissue rather than merely prevent infection. Furthermore, the capabilities of the physiologic human airway tissue chip should be harnessed to assess the effectiveness of amodiaquine/desethylamodiaquine in treating SARS-CoV-2-infected cells. These types of tests will establish the significance of physiologic tissue chip in repurposed drug screens and potentially lower the barrier for a drug to reach human clinical trials for SARS-CoV-2 treatment.

Reference Hoffmann, M., Mösbauer, K., Hofmann-Winkler, H. et al. Chloroquine does not inhibit infection of human lung cells with SARS-CoV-2. Nature (2020). https://doi.org/10.1038/s41586-020- 2575-3. 